\title{
Electrical modeling of the cell-electrode interface for recording neural activity from high-density microelectrode arrays
}

\author{
Neil Joye*, Alexandre Schmid, Yusuf Leblebici \\ EPFL, STI-IEL-LSM, Bldg ELD Station 11, 1015 Lausanne, Switzerland
}

\section{A R T I C L E I N F O}

\section{Article history:}

Received 30 October 2008

Received in revised form

28 August 2009

Accepted 1 September 2009

Communicated by C.J. Wilson

Available online 25 September 2009

Keywords:

Cell-electrode electrical model

Extracellular signal recording

High-density microelectrode array

Subcellular resolution

Three-dimensional tip electrode

\begin{abstract}
A B S T R A C T
Accurate electrical models are needed to support the design of modern microelectrode arrays. The point-contact model is presented thoroughly, and an area-contact model is analytically derived in order to model the electrical characteristics of the cell-electrode interface at subcellular resolution. An optimum electrode diameter for recording the electrical activity of neurons is analytically determined at $8 \mu \mathrm{m}$, with a cell diameter of $10 \mu \mathrm{m}$ and a typical load capacitance of $10 \mathrm{pF}$. Finally, three-dimensional tip electrodes are characterized using the area-contact model. An improvement of the electrical coupling up to $20 \mathrm{~dB}$ is observed for small electrodes, in simulation.
\end{abstract}

(c) 2009 Elsevier B.V. All rights reserved.

\section{Introduction}

Microelectrode arrays (MEAs) have become an essential tool both in fundamental and applied electrophysiology research, enabling stepping from the observation of the electrical behavior of single neurons toward the simultaneous analysis of population of neural cells [26]. Commercial MEAs usually contain approximately 60 electrodes, with electrode sizes ranging from 10 to $50 \mu \mathrm{m}$, and inter-electrode spacing ranging up to $100 \mu \mathrm{m}[9,21]$. These dimensions are much larger than the $10 \mu \mathrm{m}$ typical size of vertebrate neurons used during electrophysiological experiments, and constitute a limitation in terms of spatial resolution. The accuracy of real-time discrimination of a firing neuron within a culture suffers from this spatial under-sampling, and fully relates on digital post-processing. This issue has recently been recognized, and a new generation of active, silicon-based MEAs containing a high density and a large number (in the order of several thousand) of electrodes has been proposed $[10,13,20]$. These new devices have a pitch dimension as low as $7.8 \mu \mathrm{m}$ with an electrode diameter of $4.5 \mu \mathrm{m}$ [10], enabling electrophysiological experiments at subcellular resolution.

The development of active MEAs, where CMOS microelectronic circuits are embedded along with the electrode layer into a singlechip, demands accurate compact electrical models of the interface

\footnotetext{
* Corresponding author. Tel.: +41216936927.

E-mail addresses: neil.joye@epfl.ch, neil.joye@a3.epfl.ch (N. Joye).
}

to be available, in a format enabling co-simulation. The pointcontact model [12], which has been the standard model used to describe the electrical characteristics of the cell-electrode interface does not provide enough accuracy to model the electrical properties of the interface at subcellular resolution, since spatial distributions of the different electrical characteristics at the cellelectrode interface are not modeled. An area-contact model, where the spatial distributions of the electrical characteristics are taken into account, is needed [12]. Furthermore, modern silicon fabrication technologies enable manufacturing very dense arrays of three-dimensional electrodes, for which new electrical compact models must be developed.

This paper presents analytical developments related to the electrical modeling of the cell-electrode interface in in-vitro cultured neural cells, under the new constraints caused by subcellular resolution.

A review of the point-contact model is presented. The pointcontact model has been adapted, and theoretical results are presented both in analytical, and simulation forms in Section 2. A rigorous analytical model of the area-contact model, enabling accurate simulations of high-density MEAs in recording mode has been developed, and is presented in Section 3, where the model, its analytical derivation and simulation results are shown. Finally, the area-contact model is adapted to support three-dimensional tip electrodes, enabling theoretical performance comparisons with flat electrodes to be presented in Section 4. A discussion of the results is provided in Section 5, and concluding remarks are presented as Section 6. 


\section{Point-contact model}

The point-contact model which is reviewed in this section is depicted in Fig. 1, as a system resulting of an adaptation from several publication sources, where $V_{M}$ is the electrical potential in the cell, $V_{I}$ is the voltage at the cell-electrode interface with respect to ground, and $V_{S}$ is the potential which is sensed by the amplification stage. Ground is located at a sufficiently large distance from the electrode array. This model has been widely used to describe the electrical behavior of the cell-electrode environment $[5,12,27,34]$. The assumption that the electrical potential across the cytoplasm and the bath solution is constant is made [34]. In the case of extracellular stimulation, this assumption may no longer be valid due to the large current values issued from the electrodes.

\subsection{Model of the cell membrane}

As opposed to the Hodgkin-Huxley model [18], a passive membrane is assumed. This means that no active properties of the membrane are modeled. Thus as depicted in Fig. 1, the equivalent circuit of the attached membrane consists of a resistance $R_{M}$ in parallel with a capacitance $C_{M}$ defined as

$R_{m}=\frac{1}{g_{m e m} A_{c e}}$

$C_{m}=c_{m e m} A_{c e}$

where $A_{c e}$ is the area of the attached membrane, $g_{\text {mem }}=0.3 \mathrm{mS} / \mathrm{cm}^{2}$ is the local membrane conductivity [6], and $c_{m e m}=1 \mu \mathrm{F} / \mathrm{cm}^{2}$ is the membrane capacity per unit area. In this paper, a cell with a diameter of $10 \mu \mathrm{m}$ is considered for numerical derivations. This size is typical for vertebrate neurons, such as dissociated hippocampal rat neural cells, used in electrophysiological experiments [28].

\subsection{Model of the cell-electrode interface}

The electrical equivalent circuit of the cell-electrode junction is depicted in Fig. 1 . It includes three resistances $R_{S 1}, R_{S 2}$, and $R_{S 3}$,

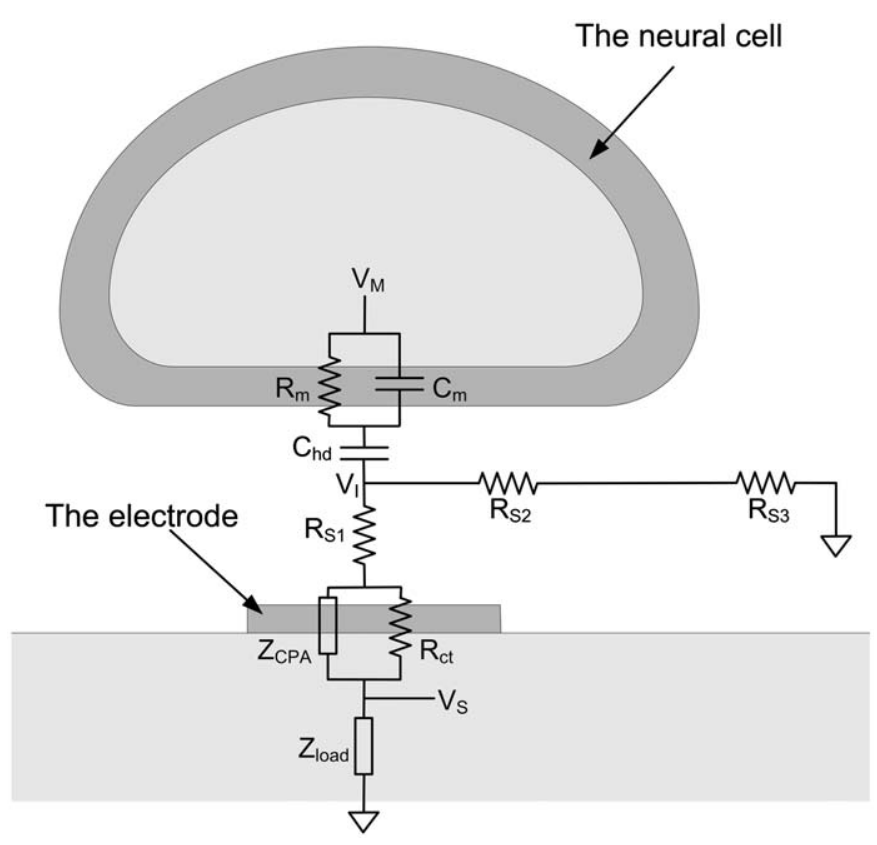

Fig. 1. Point-contact model of the cell-electrode interface (not to scale). which represent the resistance between the electrode and the counter electrode, and a cell membrane-electrolyte interface capacitance $C_{h d}$.

When no cell lies on top of the electrode, the resistance between the electrode and the counter electrode is described as

$R_{S}=R_{S 1}+R_{S 2}+R_{S 3}=R_{\text {spread }}$

where $R_{\text {spread }}$ is the spreading resistance, which is the resistance encountered by a current spreading from the electrode out into the solution. Assuming that the counter electrode is infinitely large, it is shown in [29] that the spreading resistance of a circular disk electrode with a radius $R_{e l}$ can be approximated by

$R_{\text {spread }} \cong \frac{\rho_{s}}{4 R_{e l}}$

In the case where a cell covers the entire surface of the electrode, as depicted in Fig. 1, $R_{S 2}$ is equal to the sealing resistance $R_{\text {seal }}$, which is the resistance between the cleft and the surrounding solution. This resistance has been described in [34] as

$R_{\text {seal }}=\frac{\rho_{s}}{\theta \pi d}$

where $\rho_{s}$ is the resistivity of the electrolyte (for typical solutions $\left.\rho_{s}=1 \Omega \mathrm{m}\right), d$ is the average distance between the cell and the electrode, and $\theta$ is a correction factor related to geometry $(\theta=5-8)$ $[4,5,34]$.

In this work, the potential across the electrolyte solution is considered to be constant [34]. Thus, $R_{S 3}$ can be neglected in numerical simulations. Furthermore, $R_{S 1}$ is also neglected. As shown in (3), $R_{S 1}$ is smaller or equal to $R_{\text {spread. }}$ Moreover, the electrode radius $R_{e l}$ is usually 2-3 orders of magnitude larger than the average distance $d$ between the cell and the electrode, as described in Section 2.4. Thus, $R_{\text {spread }}$ is significantly smaller than $R_{\text {seal }}$ and $R_{S 1}$ can be neglected. This assumption has been verified in simulation.

$C_{h d}$ (cell membrane-electrolyte interface capacitance) models the charge region, also called the electrical double layer, which is formed in the electrolyte at the interface with a neural cell. This electrochemical reaction appears at any interface between two different phases [19]. From the triple-layer model [3,36], the capacitance $C_{h d}$ is defined as the series of three capacitances as described in (6)

$\frac{1}{C_{h d}}=\frac{1}{C_{h 1}}+\frac{1}{C_{h 2}}+\frac{1}{C_{d}}$

where $C_{h 1}$ represents the capacitance between the membrane surface and the first-layer of non-hydrated ions (the inner Helmholtz plane); $C_{h 2}$ represents the capacitance between the layer of non-hydrated ions and the second layer of hydrated ions (the outer Helmholtz plane); and $C_{d}$ is the diffuse layer capacitance (also known as the Gouy-Chapman capacitance). These three capacitances $C_{h 1}, C_{h 2}$ and $C_{d}$ are defined as

$C_{h 1}=\frac{\varepsilon_{0} \varepsilon_{I H P}}{d_{I H P}} A_{c e}$

$C_{h 2}=\frac{\varepsilon_{0} \varepsilon_{O H P}}{d_{O H P}-d_{I H P}} A_{c e}$

$C_{d}=\frac{q \sqrt{2 \varepsilon_{0} \varepsilon_{d} k T z^{2} n^{0} N}}{k T} A_{c e}$

where $\varepsilon_{0}$ is the dielectric permittivity of free space; $\varepsilon_{I H P}$ and $\varepsilon_{O H P}$ are, respectively, the inner and outer Helmholtz planes relative dielectric constant; $d_{I H P}$ is the distance of the inner Helmholtz plane (IHP) to the cell membrane; $d_{O H P}$ is the distance of the outer Helmholtz plane (OHP) to the cell membrane; $\varepsilon_{d}$ is the diffuse layer relative dielectric constant; $k$ is Boltzmann's constant; $T$ is the absolute temperature; $q$ is the electron charge; $z$ is the valence 
of ions in solution; $n^{0}$ is the bulk concentration of ions in solution; and $N$ is Avogadro's number.

\subsection{Model of the electrode}

The equivalent circuit of the electrode is based on an electrode model typically used to describe the electrode-electrolyte impedance as depicted in Fig. 1 [11,32]. It includes a constantphase angle impedance $Z_{C P A}$, which represents the interface capacitance, in parallel with a charge transfer resistance $R_{c t}$. The Warburg impedance due to diffusion of the chemical reactants in solution, which would appear in series with $R_{c t}$, is not included in this model. It is negligible for the materials and frequency range used in electrophysiological experiments.

Using a constant-phase angle impedance $Z_{C P A}$ instead of a capacitance results in a better agreement of the model with impedance measurements [8]. Thus, $Z_{C P A}$ can be described as

$Z_{C P A}=\frac{1}{\left(j \omega C_{d l}\right)^{n}}$

where $C_{d l}$ is the double layer capacitance as described in (6)-(9), and $n$ is an empirical factor between 0 and 1 representing the surface irregularities.

The charge-transfer resistance $R_{c t}$ represents the faradic process where charges transfer between the electrode and the electrolyte by means of oxidation-reduction reactions. For small values of overpotential during a faradic charge transfer, $R_{c t}$ may be expressed as

$R_{c t}=\frac{k T}{q} \frac{1}{z J_{0} A_{e l}}$

where $z$ is the number of electrons involved in the oxidationreduction reaction, $J_{0}$ is the equilibrium exchange current density, and $A_{e l}$ is the electrode surface.

The resistance of the metal wire connecting the electrode to the circuitry and parasitic circuit elements such as fringe capacitances of this wire are neglected.

\subsection{Transfer function of the system}

The cell-electrode system depicted in Fig. 1 is described with the following equations:

$\left[V_{M}(j \omega)-V_{I}(j \omega)\right]=I_{m}(j \omega) Z_{m}(j \omega)$

$V_{I}(j \omega)=I_{\text {seal }}(j \omega) R_{\text {seal }}(j \omega)$

$\left[V_{I}(j \omega)-V_{S}(j \omega)\right]=I_{e l}(j \omega) Z_{e l}(j \omega)$

$V_{S}(j \omega)=I_{e l}(j \omega) Z_{\text {load }}(j \omega)$

$I_{m}(j \omega)=I_{\text {seal }}(j \omega)+I_{e l}(j \omega)$

where $I_{m}$ is the current flowing through the membrane; $I_{\text {seal }}$ is the current flowing through $R_{\text {seal }} ; I_{e l}$ is the current flowing through the electrode; $Z_{m}$ is the membrane impedance with $C_{h d} ; Z_{e l}$ is the electrode impedance; and $Z_{\text {load }}$ is the load impedance. The load impedance represents the input impedance of the first amplification stage. It is typically a $1-20 \mathrm{pF}$ capacitance $[2,7,15,30,38]$.

Thus, considering (12)-(16), the transfer function $H(j \omega)=$ $V_{S}(j \omega) / V_{M}(j \omega)$ of the system is established as described in the following equations:

$H(j \omega)=\frac{R_{\text {seal }} Z_{\text {load }}}{R_{\text {seal }} Z_{m}+\left(Z_{m}+R_{\text {seal }}\right)\left(Z_{\text {load }}+Z_{\text {el }}\right)}$

$Z_{m}(j \omega)=\frac{1+j \omega R_{m}\left(C_{m}+C_{h d}\right)}{\left(1+j \omega R_{m} C_{m}\right)\left(j \omega C_{h d}\right)}$
$Z_{e l}(j \omega)=\frac{R_{c t}}{1+R_{c t}\left(j \omega C_{d l}\right)^{n}}$

The Bode plots in amplitude and phase of the transfer function have been determined in Matlab, using the numerical values detailed in the following. For $C_{h d}$, the values given in [27] are considered. In particular, it is assumed that $\varepsilon_{I H P}=6, \varepsilon_{O H P}=32$, $d_{I H P}=0.3 \mathrm{~nm}, d_{O H P}=0.7 \mathrm{~nm}, z=1$, and $n^{0}=150 \mathrm{mM}$. Considering a Pt electrode, the values given in [11] are considered for $Z_{C P A}$ and $R_{C t}$; $d_{O H P}$ is equal to $0.5 \mathrm{~nm}, d_{I H P}$ is neglected, $z=4, n^{0}=154 \mathrm{mM}$, and $n=0.9$. Furthermore, the relative dielectric permittivity $\varepsilon_{r}$ of the electrolyte solution at the interface can take values between 6 and $78.54[3,25] . \varepsilon_{r}=50$ is accepted as a typical value [25]. Finally a typical load capacitance of $10 \mathrm{pF}$ is taken into account.

The Bode plots for the amplitude and phase of the transfer function are determined for a typical cell-electrode distance of $70 \mathrm{~nm}$ [27] and a circular electrode with a diameter of $5 \mu \mathrm{m}$, as depicted in Fig. 2. The value of the scaling factor $\theta$ used to determine the sealing resistance in $(5)$ is fixed to 5.78 as described in $[4,5]$. The voltage drop across the electrode is almost negligible in the frequency range of $10 \mathrm{~Hz}-10 \mathrm{kHz}$, which corresponds to the considered frequency range of neural activity. Moreover for this
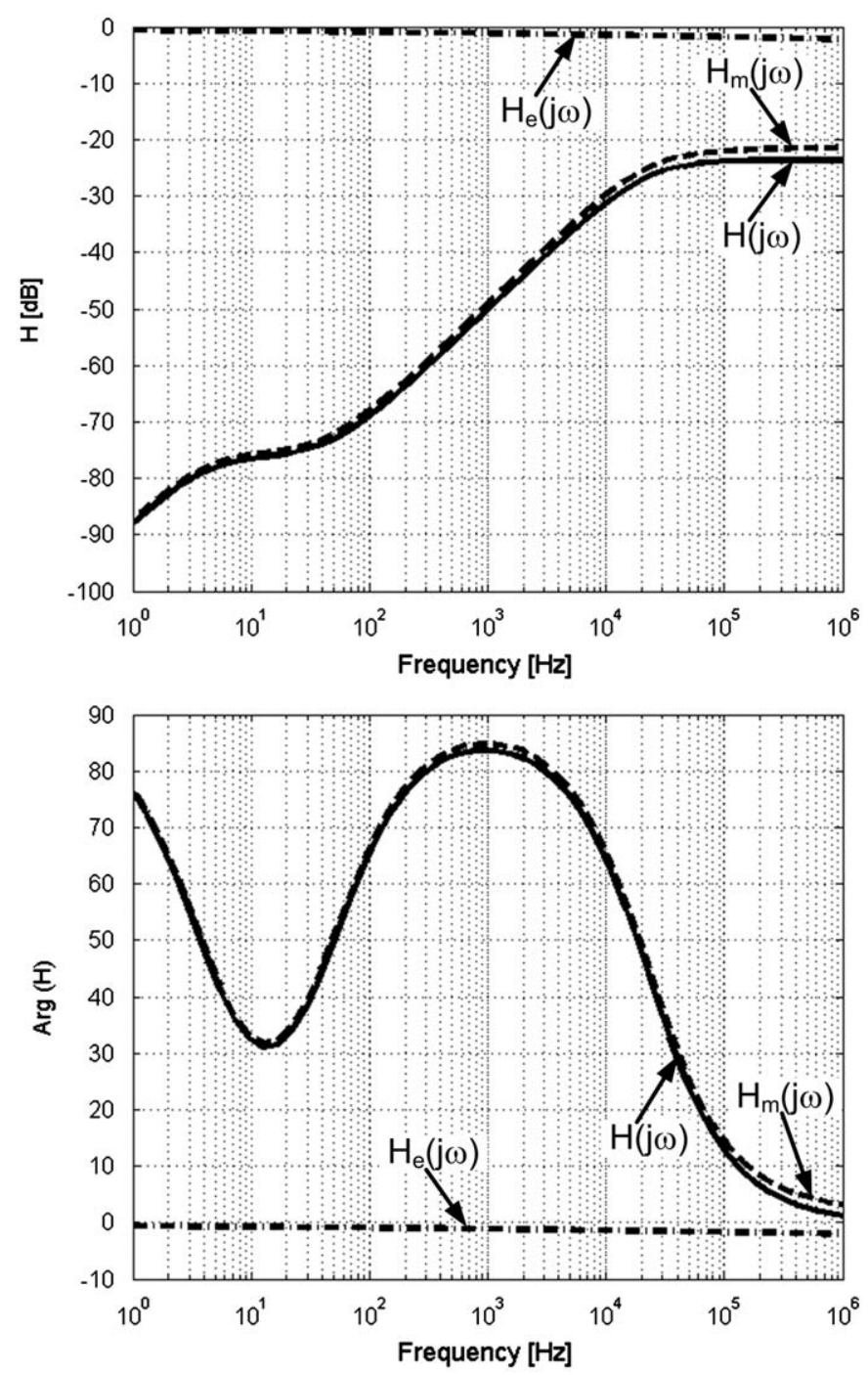

Fig. 2. Bode plots for the amplitude and phase of the transfer function $H(j \omega)$ of the system. A cell-electrode distance of $70 \mathrm{~nm}$ and a circular electrode with a diameter of $5 \mu \mathrm{m}$ are considered. $H(j \omega)=H_{m}(j \omega) H_{e}(j \omega)=V_{S}(j \omega) / V_{M}(j \omega)$ is the transfer function of the system, with $H_{m}(j \omega)=V_{I}(j \omega) / V_{M}(j \omega)$ and $H_{e}(j \omega)=V_{S}(j \omega) / V_{I}(j \omega)$. 
frequency range, the voltage attenuation between the potential that is sensed $V_{S}(j \omega)$ and the intracellular potential $V_{M}(j \omega)$ has a large variation in amplitude depending on the frequency of the neural signal. In the configuration chosen for Fig. $2, V_{S}(j \omega)$ is about 4 orders of magnitude smaller than $V_{M}(j \omega)$ for a frequency of $10 \mathrm{~Hz}$, and only 2 orders of magnitude smaller for a frequency of $3 \mathrm{kHz}$. Therefore, in the frequency range of interest, the cell-electrode system behaves as a high-pass filter. This latter characteristics is also presented in [14,31,39].

When designing a MEA, the only parameters that can be modified in the point-contact model described in Fig. 1 are the size and shape of the electrode (the load capacitance is supposed to be fixed). Therefore considering a circular electrode, the amplitude of the transfer function $H(j \omega)$ versus the electrode radius is derived in Fig. 3. The assumption that the whole electrode surface is covered by the neuron is made. If this is not the case, and as described in [27], a surface overlapping coefficient that takes into account the contact area between the cell and the electrode has to be taken into account when specifying the different elements in the point-contact model. As expected, the size of the electrode must be large enough in order to obtain a sufficiently small voltage drop between $V_{S}(j \omega)$ and $V_{M}(j \omega)$. As observed in Fig. 3, the transfer function $H(j \omega)$ drops dramatically for an electrode radius smaller than $1 \mu \mathrm{m}$. Furthermore, for diameters larger than $5 \mu \mathrm{m}$, increasing the electrode size has almost a negligible effect on the voltage drop.

Two parameter variations are considered in Fig. 3, resulting in a variability interval graphically depicted with vertical bounded segments. First, the relative dielectric permittivity $\varepsilon_{r}$ of the electrode interface is initially set to 50 , as justified above. However, $\varepsilon_{r}$ can be as low as 6 at the interface if oriented water molecules in the diffuse layer are assumed, and as large as 78.54 in the case of water at $25^{\circ} \mathrm{C}[3,25]$. Secondly, the factor $n$ in (10), which represents the electrode surface irregularities, is initially set to 0.9 . However, it has been shown that this value varies approximately between 0.87 and 0.93 for Pt electrodes [11,35]. Thus for the worst case, where $\varepsilon_{r}=6$ and $n=0.93$, the amplitude of the transfer function can be as much as $20 \mathrm{~dB}$ smaller than the

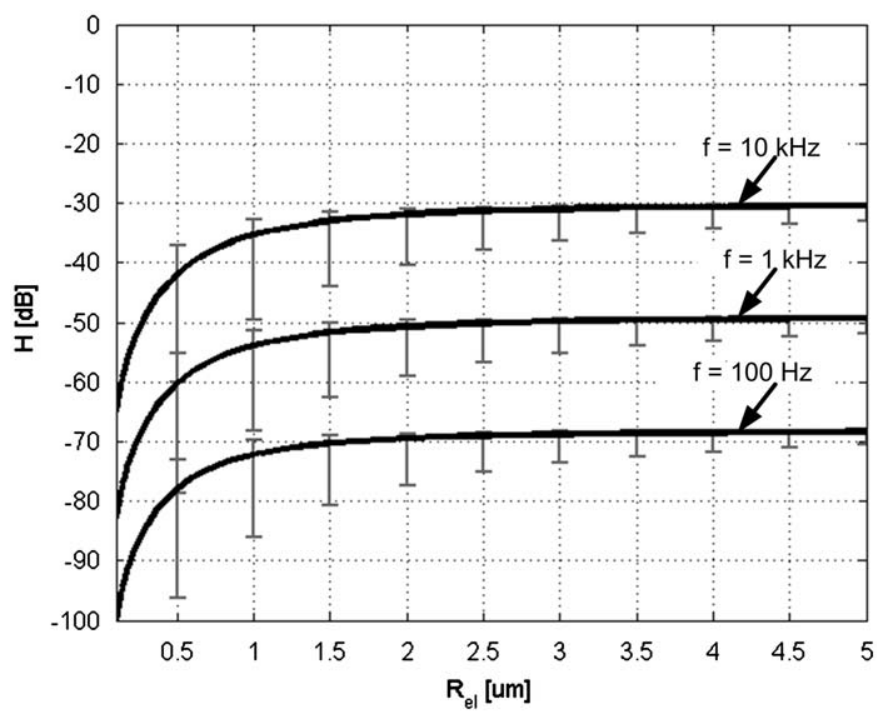

Fig. 3. Amplitude of the transfer function $H(j \omega)$ versus the electrode radius for different frequencies of $V_{M}(100 \mathrm{~Hz}-10 \mathrm{kHz})$ corresponding to the frequency range of neural activity. A cell-electrode distance of $70 \mathrm{~nm}$ and a neural cell diameter of $10 \mu \mathrm{m}$ are considered. Expected limits of variability for $\varepsilon_{r}$ (between $[6,78]$ ) and $n$ (between $[0.87,0.93]$ ) are indicated with vertical bars. Plots are produced with $\varepsilon_{r}=50, n=0.9$. typical case at subcellular resolution. For larger electrodes, this incertitude is less significant. Variations of other parameters, such as the resistivity of the electrolyte $\rho_{s}$ or the equilibrium exchange current density of the electrode $J_{o}$, could also be considered. However, their effects are negligible.

\section{Area-contact model}

An area-contact model [12], where the spatial distribution of the electrical characteristics is taken into account, is presented in this section. The goal is to describe the electrical properties of the cell-electrode interface at subcellular level when recording the electrical activity of neural cells. As for the point-contact model, the assumption that the electrical potential across the cytoplasm and the bath solution is constant is made. Future models must also include the impact of local action potential currents on the spatial distribution of intracellular potential at the subcellular scale, which is beyond the scope of this paper. The neural cell is considered to have a typical diameter size of $10 \mu \mathrm{m}$.

\subsection{Cell-electrode interface voltage}

First, the voltage $V_{I}(r, j \omega)$ at the cell-electrode interface, where $r$ is the distance to the center of the cell, is established. As a first approximation step, the electrode is not taken into account. Thus, the equivalent circuit of the attached membrane is described in Fig. 4 with the following circuit element parameters:

$$
\begin{aligned}
& r_{m}(r)=\frac{1}{g_{m e m} \partial A_{c e}} \\
& c_{m}(r)=c_{m e m} \partial A_{c e} \\
& c_{h}(r)=\frac{C_{h d}}{A_{c e}} \partial A_{c e}=c_{h d} \partial A_{c e} \\
& \partial A_{c e}=2 \pi r \partial r \\
& r_{\text {seal }}(r)=\frac{\rho}{d} \frac{\partial r}{2 \pi r}
\end{aligned}
$$

In the Laplace domain, the attached membrane can be described as

$\frac{V_{I}(r, s)-V_{I}(r-\partial r, s)}{r_{\text {seal }}(r-\partial r)}+\frac{V_{M}(s)-V_{I}(r, s)}{z_{m}(r)}=\frac{V_{I}(r+\partial r, s)-V_{I}(r, s)}{r_{\text {seal }}(r)}$

With the approximation given in (26) and the circuit elements described in (20)-(24), the cell-electrode interface can be expressed as the first order linear equation given in (27)

$$
\begin{aligned}
& \partial V_{I}(r, s)=V_{I}(r, s)-V_{I}(r-\partial r, s)=V_{I}(r+\partial r, s)-V_{I}(r, s) \\
& \frac{\partial V_{I}(r, s)}{\partial r}+2 \operatorname{arV}_{I}(r, s)=2 \operatorname{arV}_{M}(s)
\end{aligned}
$$

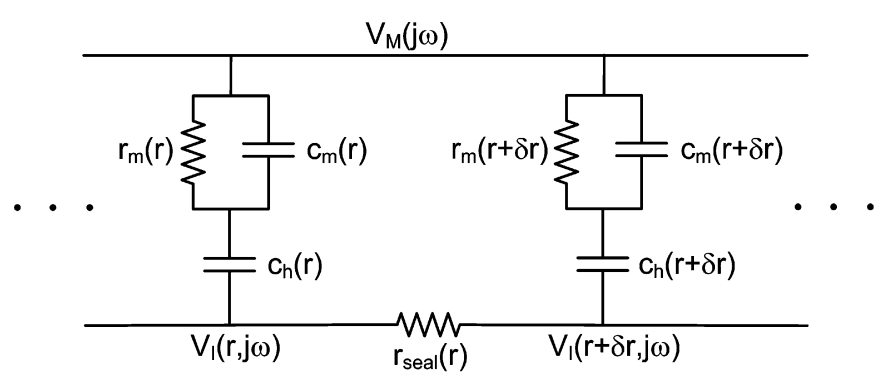

Fig. 4. Area-contact model of the attached cell membrane, where $V_{M}(j \omega)$ is the intracellular potential, and $V_{I}(r, j \omega)$ is the potential at the cell-electrode interface at a distance $r$ from the center of the cell. 
where $a$ is

$a=\frac{s \rho_{s} c_{h d}\left[g_{m e m}+s c_{m e m}\right]}{2 d\left[g_{m e m}+s\left(c_{m e m}+c_{h d}\right)\right]}$

The voltage $V_{I}\left(R_{c e}, S\right)$ at the border of the neural cell, where $R_{c e}$ is the radius of the cell, is considered to be equal to the potential of the bath solution [4]. Thus with the initial condition $V_{I}\left(R_{c e}, S\right)=0$, the solution to this equation is as follows:

$V_{I}(r, s)=V_{M}(s)\left(1-e^{a\left(R_{c e}^{2}-r^{2}\right)}\right)$

The distribution of the cell-electrode interface voltage $V_{I}(r, s)$ for various frequencies in the range of neural activity is shown in Fig. 5. The electrical property values that were used for the pointcontact simulation depicted in Fig. 2 are considered as well. As expected, $V_{I}(r, s)$ is maximum at the center of the cell and is minimum at the edge of the cell.

\subsection{Transfer function of the system for an electrode centered with} the cell

A circular electrode is now included into the area-contact model. Thus, with a first approximation where the spreading resistance is neglected, the electrode can be modeled as presented

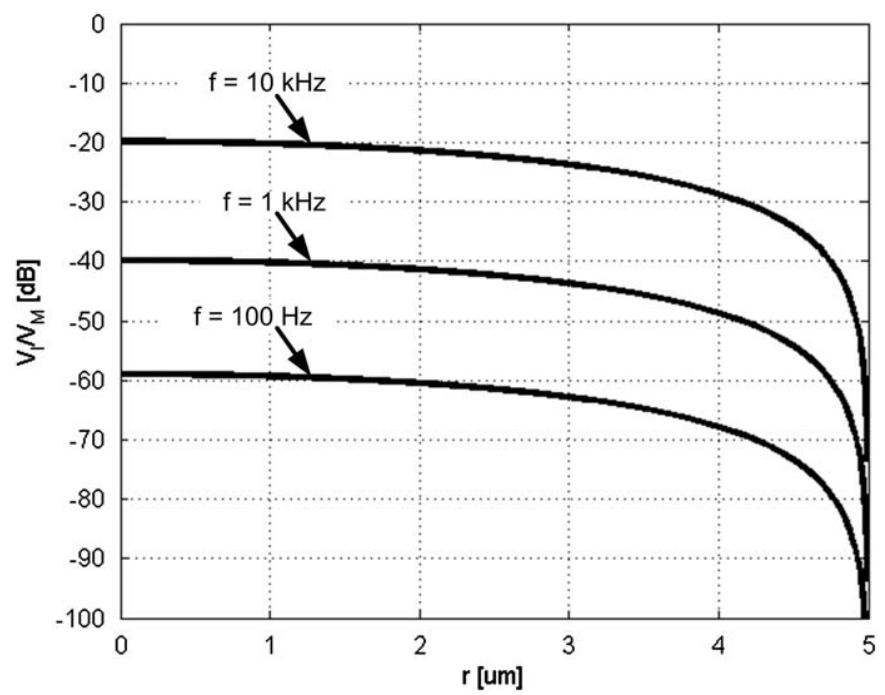

Fig. 5. $V_{I} / V_{M}$ versus the distance from the center of the cell for different frequencies of $V_{M}(100 \mathrm{~Hz}$ to $10 \mathrm{kHz})$ corresponding to the frequency range of neural activity. A cell-electrode distance of $70 \mathrm{~nm}$ is considered.

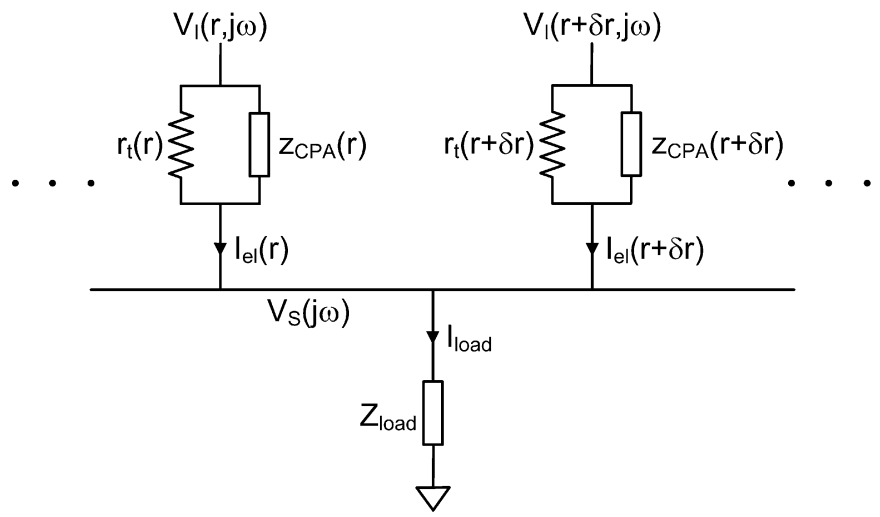

Fig. 6. Area-contact model of the electrode with the load impedance. in Fig. 6. The definition of the circuit elements is as follows:

$r_{t}(r)=R_{c t} \frac{A_{e l}}{\partial A_{e l}}=\frac{r_{c t}}{\partial A_{e l}}$

$z_{C P A}(r)=\frac{1}{\left(j \omega c_{d}\right)^{n}}$

$c_{d}(r)=\frac{C_{d l}}{A_{e l}} \partial A_{e l}=c_{d l} \partial A_{e l}$

where $r_{c t}$ and $c_{d l}$ are, respectively, the area specific values of the charge-transfer resistance and double layer capacitance.

Furthermore if the current absorbed by the electrode is neglected, which is the case for frequencies smaller than $10 \mathrm{kHz}$, $V_{S}(s)$ can be expressed as

$V_{s}(s)=Z_{\text {load }} \int_{0}^{R_{e l}} \frac{V_{I}(r, s)-V_{s}(s)}{Z_{e l}(r, s)}$

where $z_{e l}(r, s)$ consists of $r_{c}(r)$ in parallel with $z_{C P A}(r)$.

As for the point-contact model, the load impedance is considered to be a capacitance $C_{\text {load }}$ of $10 \mathrm{pF}$. Therefore using expressions (28)-(33), $V_{S}(s)$ can be expressed as

$V_{s}(s)=\frac{\pi\left(1+r_{c t}\left(s c_{d l}\right)^{n}\right)}{s C_{\text {load }} r_{c t}+R_{e l}^{2} \pi\left(1+r_{c t}\left(s c_{d l}\right)^{n}\right)}\left[R_{e l}^{2}+\frac{e^{a\left(R_{c e}^{2}-R_{e l}^{2}\right)}-e^{a R_{c e}^{2}}}{a}\right] V_{M}(s)$

The transfer function of the cell-electrode system $H(s)=V_{S}(s)$ / $V_{M}(s)$ versus the electrode radius is presented in Fig. 7(a) for different frequencies of $V_{M}(s)$. The physical and electrical parameter values that were used in Figs. 2 and 5 are considered again. As shown in these graphs, for electrodes diameters smaller than $1 \mu \mathrm{m}$, the amplitude of $H(s)$ can be more than $20 \mathrm{~dB}$ smaller than it is in the case of electrode diameters around $8-10 \mu \mathrm{m}$. Consequently, larger electrode sizes should be considered, when mechanical limitations permit it. However, if the electrode size is too large, $V_{S}(s)$ slightly decreases due to the decrease of $V_{I}(r, s)$ at the edge of the cell as depicted in Fig. 5. An optimum electrode radius can be clearly identified at approximately $4 \mu \mathrm{m}$ for the typical case.

As described in Fig. 7(a), the frequency has negligible effects on the optimum electrode size. Similar results have been found regarding the cell-electrode distance [23]. However, this optimum size has a strong dependence on the load capacitance, as described in Fig. 7(b). The larger the load capacitance, the larger is the optimum electrode size. Moreover, the effect of $C_{\text {load }}$ is stronger for small electrodes. This information is very useful for the design of the amplification stage of a silicon-based MEA.

As explained earlier, unlike the point-contact model, the areacontact model takes into account the voltage distribution at the interface between the cell and the electrode. This explains why $V_{S}(s)$ slightly decreases when the electrode size is increased above the optimum electrode radius. Moreover, the area-contact model also takes into account the spatial distribution of the current flowing through the electrode $I_{e l}(r)$, as depicted in Fig. 6 . The smaller dependence of the transfer function $H(s)$ derived from the point-contact model on the electrode size is determined by this phenomenon. This can be evidenced by comparing Figs. 3 and $7(a)$.

The parameter variations used in Fig. 3 are also considered in Fig. 7. As for the point-contact model, the amplitude of the transfer function has a maximum incertitude of approximately $20 \mathrm{~dB}$ for small electrode sizes. Moreover, it is shown in Fig. 7(b) that this incertitude is less significant when smaller load capacitances are used. In the worst case, where $\varepsilon_{r}=6$ and $n=0.87$, the optimum electrode radius is approximately equal to $5 \mu \mathrm{m}$. 
(a)

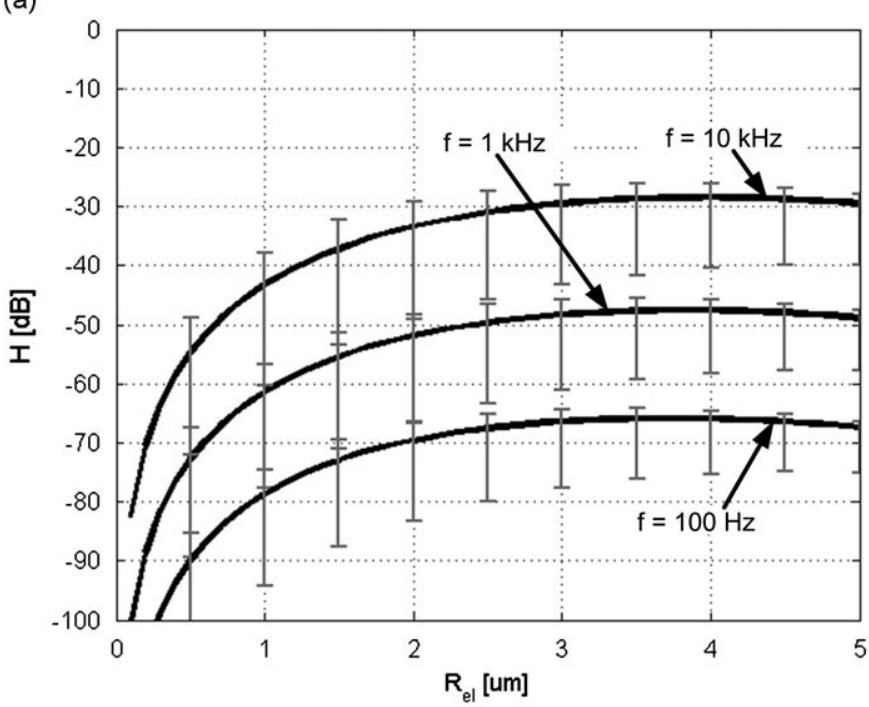

(b)

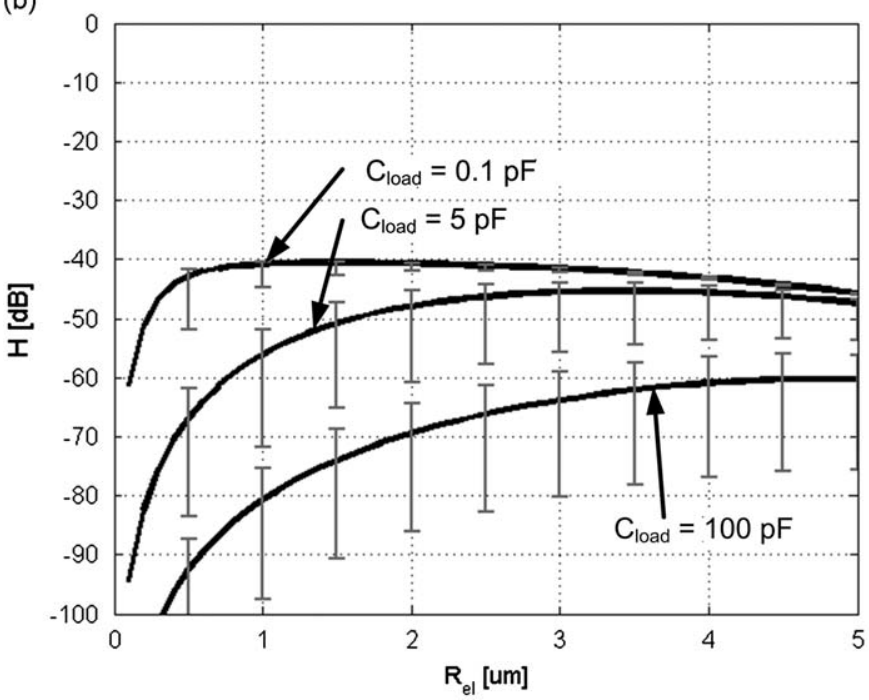

Fig. 7. Amplitude of the transfer function $H(s)=V_{S}(s) / V_{M}(s)$ versus the electrode radius for (a) different frequencies of $V_{M}$ corresponding to the frequency range of neural activity and (b) different load capacitances. A load capacitance of $10 \mathrm{pF}$ is used in (a) and a frequency of $1 \mathrm{kHz}$ is considered in (b). In both cases, a cellelectrode distance of $70 \mathrm{~nm}$ is used. Expected limits of variability for $\varepsilon_{r}$ (between $[6,78])$ and $n$ (between $[0.87,0.93]$ ) are indicated with vertical bars. Plots are produced with $\varepsilon_{r}=50, n=0.9$.

\section{Three-dimensional tip electrode}

A new approach based on three-dimensional tip electrodes has been developed as a way to increase the electrical coupling between the cultured neural cells and the individual sensors in a MEA [22]. With respect to standard planar electrodes, the electrical coupling between an electrode and a neural cell is expected to be improved as a benefit of a larger cell-electrode contact area.

As pointed out in Section 3, the area-contact model is appropriate to describe the electrical behavior of a cell-electrode interface at subcellular resolution. Thus, this model is suited to describe the electrical behavior of the proposed three-dimensional tip electrode, and is derived analytically in this section. The following set of numerical parameters has been chosen in the simulations, as realistic values which can be obtained using a modern silicon fabrication technology in the development of the (a)

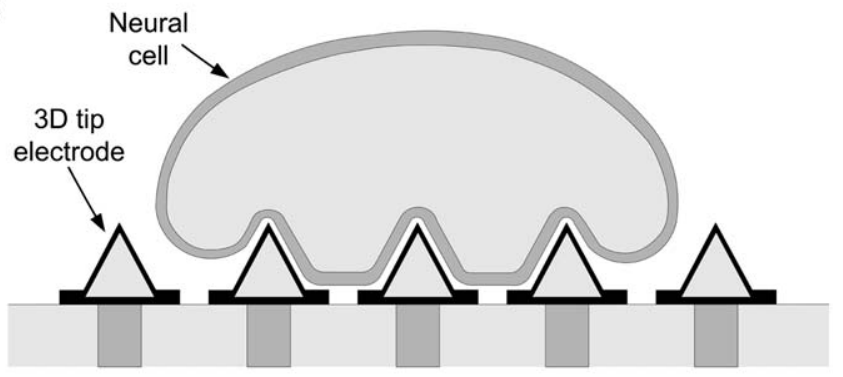

(b)

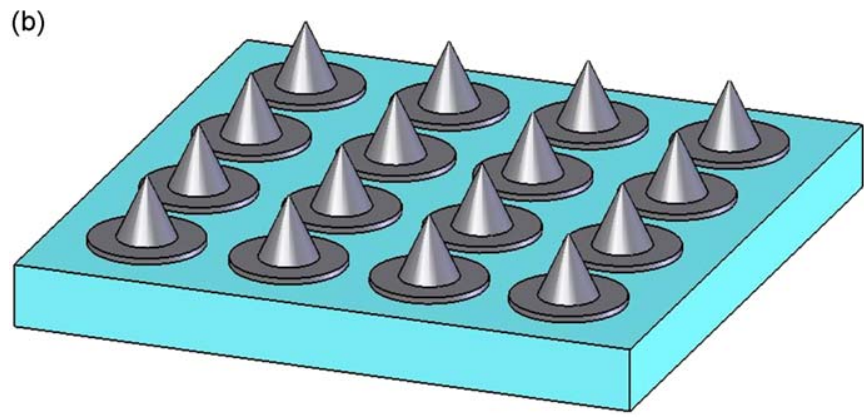

Fig. 8. (a) Conceptual cross-section of a neural cell lying on the tips of a threedimensional MEA with the attached membrane adapting to the surface topology. (b) Conceptual view of the surface of a $4 \times 4$ three-dimensional tip electrode array.

structure described in Fig. 8. Three-dimensional tip electrodes have a diameter of $3-4 \mu \mathrm{m}$, a height of $1.75 \mu \mathrm{m}$ and a pitch of 5-6 $\mu \mathrm{m}$, which guarantees that every neuron is in electrical contact with several electrodes [22], as depicted in Fig. 8.

This technology must not be confused with three-dimensional MEAs which are reported in literature in $[1,17,37]$. In these cases, the three-dimensional electrodes are used to penetrate a layer of cells which have been injured during the preparation of acute brain slices, and is located at the tissue slice border. Consequently, their heights are much more significant, and range from 47 to $100 \mu \mathrm{m}$.

\subsection{Cell-electrode interface voltage}

Following the method applied in the case of planar electrodes, the cell-electrode interface voltage $V_{I}(r, s)$ is established for a cell centered with the tip of a three-dimensional cone. As a first approximation step, the electrode is not taken into account. Thus, the equivalent circuit of the attached membrane is described in Fig. 9(a), where $z_{m}(r)$ consists of $c_{h d}(r)$ in series with $r_{m}(r) / / c_{m}(r)$ as described in Fig. $4 ; H_{c}$ is the height of the three-dimensional cone, and $R_{c}$ the radius of its base. The electrical property values that were used for the planar electrodes simulations are also considered in this section. In order to derive the cell-electrode interface voltage $V_{I}$, the axis $l$ along the three-dimensional surface is considered instead of the axis $r$, as depicted in Fig. 9(b). Thus, $V_{I}(l, s)$ can be expressed as in (29)

$$
V_{I}(l, s)=V_{M}(s)\left(1-e^{a\left(L_{c e}^{2}-l^{2}\right)}\right)
$$

Furthermore, the three-dimensional topology has an influence on the cell-electrode distance $d(l)$ as shown in Fig. 9(b). For $r>R_{c}$, the cell-electrode distance is considered to be equal to the cellelectrode distance $d_{2 D}$ of a cell lying on a flat surface. For $r<R_{c}$, $d(l)$ is expressed in function of $d_{2 D}$ and $d_{t i p}$, where $d_{t i p}$ is the cellelectrode distance at the tip of the cone as depicted in Fig. 9(b). This distance depends on the height $H_{c}$, the radius $R_{c}$, and the flexibility of the cell membrane. It is expected to be smaller than $d_{2 D}$. However, the exact value of this distance may vary since the 
(a)

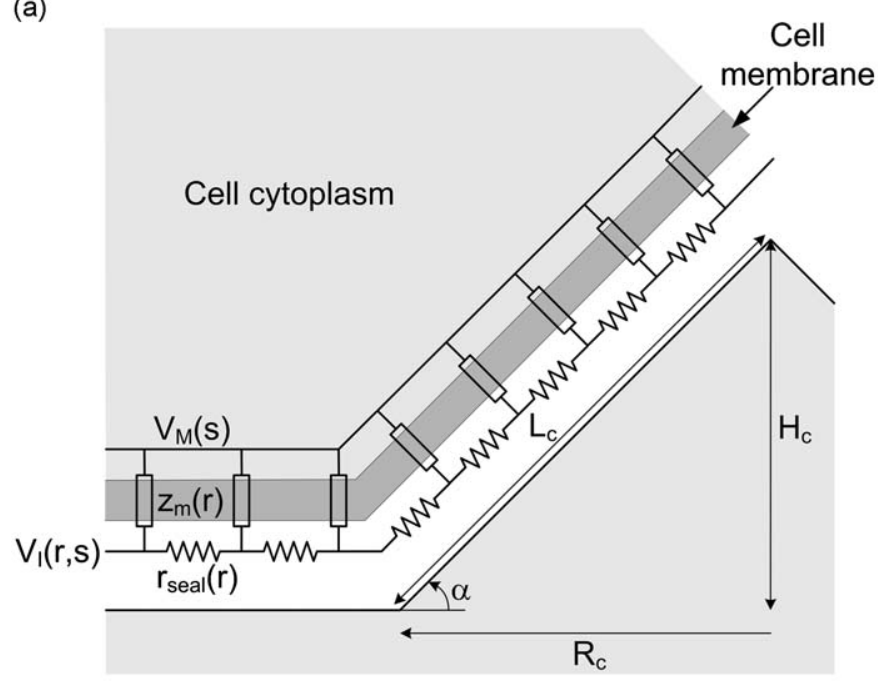

(b)

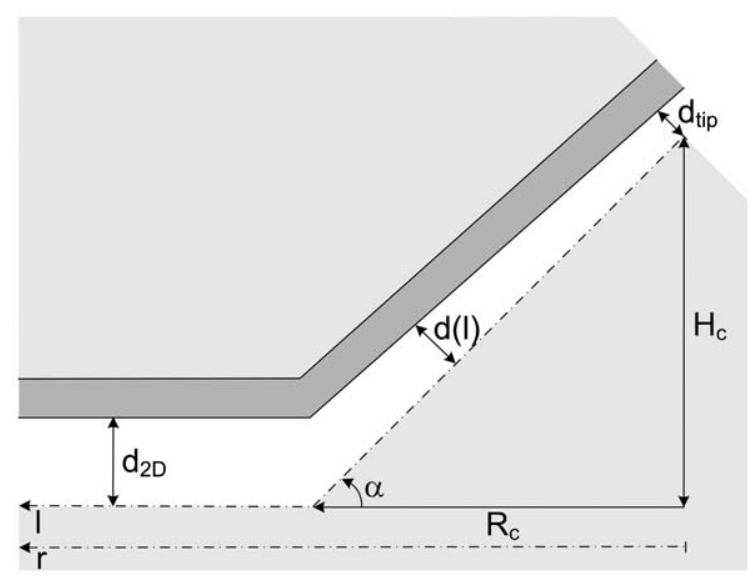

Fig. 9. (a) Area-contact model of the attached membrane of a cell lying on the tip of a three-dimensional cone; $H_{c}$ is the height of the three-dimensional cone and $R_{c}$ is the radius of its base. (b) Schematic representation of the cell-electrode distance $d(l)$ for a cell lying on the tip of a three-dimensional surface; $d_{2 D}$ is the cellelectrode distance of a cell lying on a flat surface, and $d_{\text {tip }}$ is the cell-electrode distance at the tip.

cell-electrode interface mainly consists of glycocalix and adhesion promoting proteins such as laminin or poly-d-lysine [33]. Using the above mentioned approximations, $d(l)$ is expressed as

$d(l)=\frac{d_{2 D}-d_{t i p}}{L_{c}} l-d_{t i p}$

$l=\frac{r}{\cos \alpha}$

when $r \leq R_{c} . d(r)$ is therefore equal to

$d(r)=\left(d_{2 D}-d_{\text {tip }}\right) \frac{r}{R_{c}}+d_{\text {tip }}$

If $r>R_{c}$, then $d(r)=d_{2 D}$ and

$l=R_{c}\left(\frac{1-\cos \alpha}{\cos \alpha}\right)+r$

The distribution of the cell-electrode interface voltages $V_{I}(r, s)$ for different cell-electrode distances $d_{\text {tip }}$ at the tip of the threedimensional cone is described in Fig. 10. A frequency of $1 \mathrm{kHz}$, a cell-electrode distance $d_{2 D}$ of $70 \mathrm{~nm}$, a height $H_{c}$ of $1.75 \mu \mathrm{m}$, and a radius $R_{c}$ of $1.5 \mu \mathrm{m}$ are considered.

As shown in Fig. 10, the three-dimensional tip has two effects on the cell-electrode voltage $V_{I}(r, s)$. The first one is an increase of

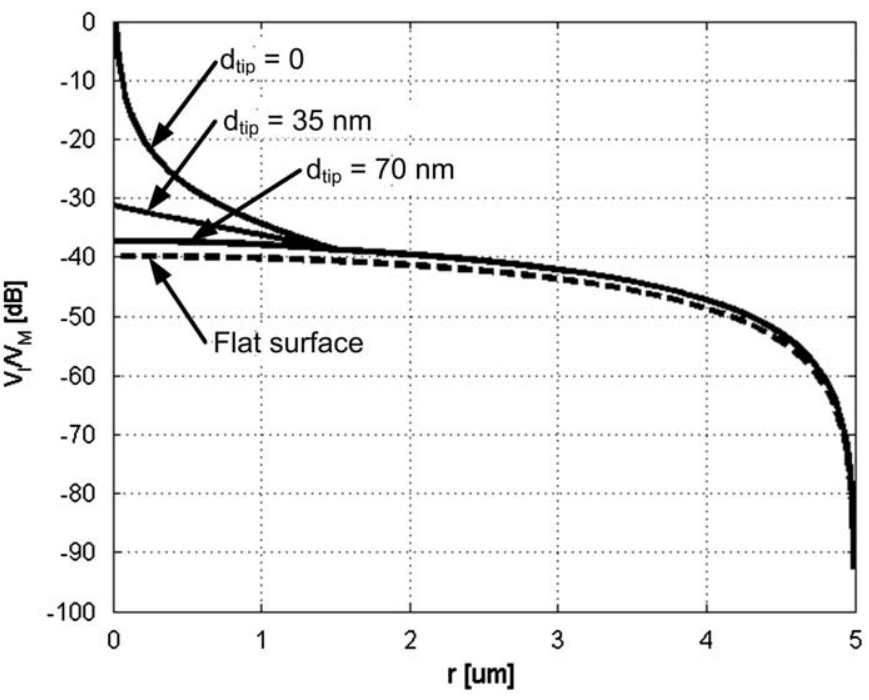

Fig. 10. $V_{I} / V_{M}$ versus the distance from the center of the cell for different cellelectrode distances $d_{\text {tip }}$ at the tip. A frequency of $1 \mathrm{kHz}$, a cell-electrode distance $d_{2 D}$ of $70 \mathrm{~nm}$, a height $H_{c}$ of $1.75 \mu \mathrm{m}$, and a radius $R_{c}$ of $1.5 \mu \mathrm{m}$ are considered.

$V_{I}(r, s)$ obtained with the three-dimensional topology compared to the one obtained with a flat surface. This increase is approximately equal to $2-3 \mathrm{~dB}$ and is due to a larger area of the attached membrane. The second effect is an increase of $V_{I}(r, s)$ for $r$ smaller than $R_{c}$. This increase is due to a decrease of the cell-electrode distance around the tip of the three-dimensional structure which increases $r_{\text {seal }}$. This increase can be as large as 30-40 dB at the tip if the cell is touching the tip of the three-dimensional surface $\left(d_{\text {tip }}=0\right)$. However, a very short distance between the electrode tip and the neuron cell is assumed in this work, de-facto resulting in a favorable situation, as explained earlier. These two effects are also observed with different frequencies between $10 \mathrm{~Hz}$ and $10 \mathrm{kHz}$ and with cell-electrode distances between 10-100 $\mathrm{nm}$.

\subsection{Transfer function of the system for a three-dimensional tip electrode centered with the cell}

A three-dimensional circular electrode is now included into the model, as depicted in Fig. 8. The electrode model presented in Fig. 6 is used with a typical load capacitance of 10 pF. Following the method applied for the determination of the cell-electrode interface voltage $V_{I}(r, s), V_{S}(s)$ can be expressed as (33) and (34) using $d$ and $l$ from (36)-(39). Thus, the transfer function of the cell-electrode system $H(s)=V_{S}(s) / V_{M}(s)$ versus the electrode radius is presented in Fig. 11 for different cell-electrode distances $d_{\text {tip }}$ at the tip. The electrical and physical parameters used in Fig. 10 are considered.

As shown in Fig. 11, using three-dimensional tip electrodes impacts on $V_{S}(s)$ in two ways, mostly. First, an increase of $V_{S}(s)$ compared to the value obtained with planar electrodes is observed. This result is due to a larger area of the attached membrane, which increases $V_{I}(s, r)$ as depicted in Fig. 10. The increase of $V_{S}(s)$ is between $3-10 \mathrm{~dB}$ depending on the size of the electrode.

Second, an increase of $V_{S}(s)$ when $d_{t i p}$ is decreased is observed for $r$ smaller than $R_{c}$. As explained above, this increase is due to the decrease of the cell-electrode distance around the tip of the three-dimensional structure which increases $r_{\text {seal }}$. This increase of $V_{S}(s)$ can be as large as $20 \mathrm{~dB}$ for an electrode diameter of $1 \mu \mathrm{m}$, as shown in Fig. 11. However, the electrode impedance is large for small electrode sizes. Therefore this effect does not influence the optimum size of the electrode. For the three-dimensional 


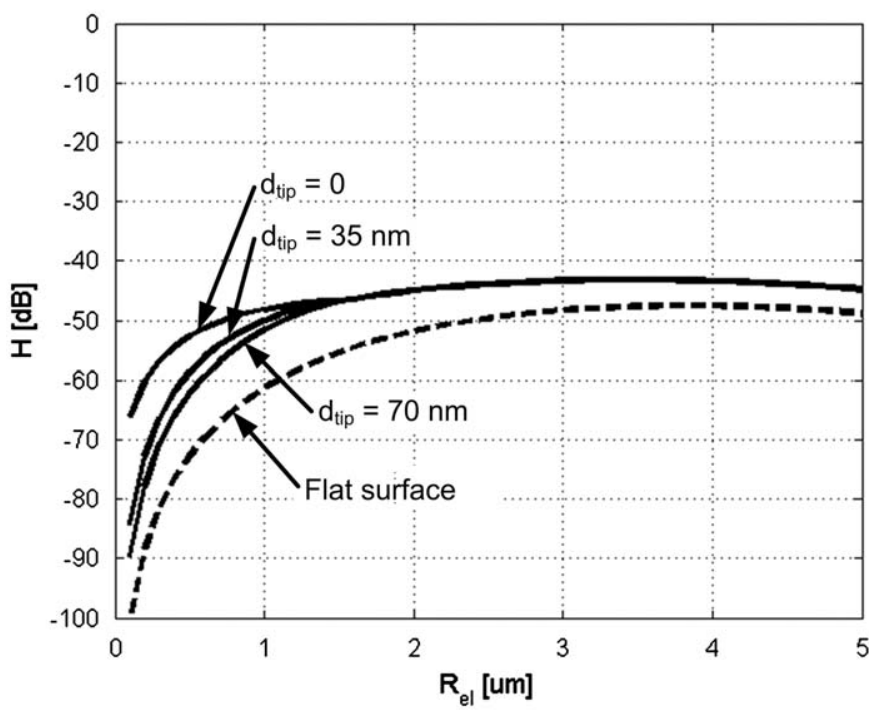

Fig. 11. Amplitude of the transfer function $H(s)=V_{S}(s) / V_{M}(s)$ versus the electrode radius for different cell-electrode distances $d_{\text {tip }}$ at the tip. A frequency of $1 \mathrm{kHz}$, a cell-electrode distance $d_{2 D}$ of $70 \mathrm{~nm}$, a height $H_{c}$ of $1.75 \mu \mathrm{m}$, and a radius $R_{c}$ of $1.5 \mu \mathrm{m}$ are considered. The load impedance is a $10 \mathrm{pF}$ capacitance.

topology and physical parameters used in this section, the optimum electrode diameter size is slightly smaller than for planar electrodes, and is identified between 6 and $7 \mu \mathrm{m}$.

The design of MEAs can largely benefit from exploiting the aforementioned results. If no severe spatial constraints are applied to the design, the optimum electrode diameter is chosen in order to obtain the maximum amplitude of the measured voltage $V_{S}(s)$. In this configuration, the three-dimensional electrode offers a $3 \mathrm{~dB}$ increase in the amplitude of the system transfer function, compared to planar electrodes. However, when designing a high-density MEA, the electrode size can be constrained by small inter-electrode spacings. Thus, a smaller electrode size has to be chosen. In this case, selecting a three-dimensional electrode could be an appropriate solution, where the increase of $V_{S}(s)$ compared to planar electrode can be has large as $10 \mathrm{~dB}$ for $d_{\text {tip }}$ equal to $d_{2 D}$ and $20 \mathrm{~dB}$ for $d_{\text {tip }}$ equal to zero.

\section{Discussion}

This paper focuses on presenting rigorous analytical expressions for three cell-electrode interfaces. The widely-accepted point-contact model is presented in a form adapted from $[11,27,34]$. The area-contact model expressions for flat and three-dimensional electrodes are presented. Electrical simulations for the three cases are presented, under various parametric conditions.

The models are presented as tools that are used to support the development of silicon-MEAs, including high-resolution MEAs $[10,13,20]$. The systematic analysis of the area-contact models demonstrates the existence of an optimum electrode size, which can be derived for planar and three-dimensional electrodes, and which depends on the load capacitance. Nevertheless, this optimum electrode size also depends on the cell type and size. In this paper, standard dissociated hippocampal rat neural cells, which are typically used in electrophysiological experiments, are considered.

The models have primarily been developed to enable the electrical co-simulation of the cell-electrode interface along with the low-noise amplifiers stages which are driving neural signals off-chip, into analog-to-digital converters, and subsequent digital processing units $[2,7,10,13,15,20,30,38]$. A noise model can be inserted into the model of the electrode, to enable realistic noise simulations of the full system [24]. Ongoing work in this direction is currently performed in order to determine the specifications of a low-noise CMOS amplifier stage (input impedance, input referred noise, etc.).

Electrical measurements to be performed on fabricated MEAs, and comparisons with the proposed models are not addressed in this paper, intentionally. However, realistic variation of electrical model parameters has been performed to determine the incertitude interval of the simulated results. In the area-contact model, the maximum incertitude in the amplitude of the transfer function reaches approximately $20 \mathrm{~dB}$ for subcellular resolution. This incertitude is shown to be less significant for larger electrodes and smaller load capacitances.

As a major theoretical result, three-dimensional tip electrodes are demonstrated to increase the electrical potential that is sensed compared to planar electrodes, using the three-dimensional adapted area-contact model. This increase is due to a larger cell-electrode contact area due to the three-dimensional topology, and a closer cell-electrode distance around the tip of the electrode. An additional increase of $V_{S}(s)$ could also be obtained using Pt-black or TiN electrodes coating (planar or threedimensional) [11]. Because of their roughness, these types of electrodes benefit from the substantial increased electrode area.

Finally, three-dimensional tip electrodes have also been designed with the target of performing electrical stimulation of neural cells. In this case, special care needs to be taken to the strong electric fields at the electrode tips in order to prevent electroporation of the neural cells [16]. Stimulation models are thus specific and different from the recording models, and are beyond the scope of this paper.

\section{Conclusion}

Analytical models enabling the electrical co-simulation of the cell-electrode interface, along with analog front-end electronics are presented in two main incarnations. A point-contact model adapted from several publication sources is presented, and an area-contact model is analytically derived, enabling to model the electrical behavior of the cell-electrode interface of neuron cultures during electrical activity recording with high-density MEAs.

The area-contact model is presented as a model more suitable for subcellular multi-electrode resolution, which is a requisite for modeling and simulating the electrical behavior of novel high-density silicon-based MEAs. The existence of an optimum electrode diameter has been analytically determined, using the latter model. When measuring the extracellular voltage potential of standard dissociated hippocampal neural cells, which have a diameter size of approximately $10 \mu \mathrm{m}$, the optimum is equal to $8-10 \mu \mathrm{m}$ for a planar electrode with a $10 \mathrm{pF}$ load capacitance. Moreover, the optimum electrode size has been shown to strongly depend on the load capacitance, which is typically the input capacitance of the amplification stage of silicon-based MEA.

The electrical model of the cell-electrode interface consisting of three-dimensional tip electrodes has also been derived analytically, and simulated using the area-contact model. Using the physical dimensions of manufactured electrodes in [22], an optimum electrode diameter of $6-7 \mu \mathrm{m}$ has been derived. A $10-20 \mathrm{~dB}$ increase in the amplitude of the system transfer function compared to planar electrodes can be achieved with small three-dimensional electrode arrays. This increase occurs thanks to an increase of the cell-electrode contact area, and an 
increase of the seal resistance due to an expected decrease of the cell-electrode distance around the tip of the three-dimensional electrode.

\section{Acknowledgments}

This work has been conducted with the support of the Swiss NSF Grant no. 205321-116780, and the EPFL STI Grant.

\section{References}

[1] J.N.Y. Aziz, R. Genov, B.L. Bardakjian, M. Derchansky, P.L. Carlen, Brain-silicon interface for high-resolution in vitro neural recording, IEEE Trans. Biomed. Circuits Syst. 1 (2007) 56-62.

[2] R.A. Blum, J.D. Ross, E.A. Brown, S.P. DeWeerth, An integrated system for simultaneous, multichannel neuronal stimulation and recording, IEEE Trans. Circuits Syst. I 54 (2007) 2608-2618.

[3] J.O.M. Bockris, A.K.N. Reddy, Modern Electrochemistry, Plenum Press, New York, 1970.

[4] D. Braun, P. Fromherz, Imaging neuronal seal resistance on silicon chip using fluorescent voltage-sensitive dye, Biophys. J. 87 (2004) 1351-1359.

[5] M. Brittinger, P. Fromherz, Field-effect transistor with recombinant potassium channels: fast and slow response by electrical and chemical interactions, App. Phys. A 81 (2005) 439-447.

[6] J.R. Buitenweg, W.L.C. Rutten, E. Marani, Geometry-based finite-element modeling of the electrical contact between a cultured neuron and a microelectrode, IEEE Trans. Biomed. Eng. 50 (2003) 501-509.

[7] W. Dabrowski, P. Grybos, A.M. Litke, A low noise multichannel integrated circuit for recording neuronal signals using microelectrode arrays, Biosens. Bioelectron. 19 (2004) 749-761.

[8] R.W. de Boer, A. van Oosterom, Electric properties of platinum electrodes: impedance measurements and time-domain analysis, Med. Biol. Eng. Comput. 16 (1978) 1-10.

[9] U. Egert, B. Schlosshauer, S. Fennrich, W. Nisch, M. Fejtl, T. Knott, T. Müller, H. Hämmerle, A novel organotypic long-term culture of the rat hippocampus on substrate-integrated multielectrode arrays, Brain Res. Protocols (1998) 229-242.

[10] B. Eversmann, M. Jenkner, F. Hofmann, C. Paulus, R. Brederlow, B. Holzapfl, P. Fromherz, M. Merz, M. Brenner, M. Schreiter, R. Gabl, K. Plehnert, M. Steinhauser, G. Eckstein, D. Schmitt-Landsiedel, R. Thewes, A $128 \times 128$ CMOS biosensor array for extracellular recording of neural activity, IEEE J. SolidState Circuits 38 (2003) 2306-2317.

[11] W. Franks, I. Schenker, P. Schmutz, A. Hierlemann, Impedance characterization and modeling of electrodes for biomedical applications, IEEE Trans. Biomed. Eng. 52 (2005) 1295-1302.

[12] P. Fromherz, Neuroelectronic interfacing: semiconductor chips with ion channels, nerve cells, and brain, in: R. Waser (Ed.), Nanoelectronics and Information Technology, Wiley-VCH, Berlin, 2003, pp. 781-810.

[13] U. Frey, F. Heer, R. Pedron, S. Hafizovic, F. Greve, J. Sedivy, K.-U. Kirstein, A. Hierlemann, An 11k-electrode 126-channel high-density microelectrode array to interact with electrogenic cells, in: Proceedings of IEEE ISSCC 2007, pp. 158-593.

[14] M. Grattarola, S. Martinoia, Modeling the neuron-microtransducer junction: from extracellular to patch recording, IEEE Trans. Biomed. Eng. 40 (1993) 35-41.

[15] R.R. Harrison, C. Charles, A low-power low-noise CMOS amplifier for neural recording applications, IEEE J. Solid-State Circuits 38 (2003) 958-965.

[16] J. Held, J. Gaspar, P.J. Koester, C. Tautorat, A. Cismak, A. Heilmann, W. Baumann, A. Trautmann, P. Ruther, O. Paul, Microneedle arrays for intracellular recording applications, in: Proceedings of IEEE MEMS 2008, pp. 268-271.

[17] M.O. Heuschkel, M. Fejtl, M. Raggenbass, D. Bertrand, P. Renaud, A threedimensional multi-electrode array for multi-site stimulation and recording in acute brain slices, J. Neurosci. Methods 114 (2002) 135-148.

[18] A.L. Hodgkin, A.F. Huxley, A quantitative description of membrane current and its application to conduction and excitation in nerve, J. Physiol. 117 (1952) 500-544.

[19] R.J. Hunter, Foundations of Colloid Science, Oxford University Press, New York, 2001.

[20] K. Imfeld, S. Neukom, A. Maccione, Y. Bornat, S. Martinoia, P.-A. Farine, M. Koudelka-Hep, L. Berdondini, Large-scale, high-resolution data acquisition system for extracellular recording of electrophysiological activity, IEEE Trans. Biomed. Eng. 55 (2008) 2064-2073.

[21] Y. Jimbo, N. Kasai, K. Torimitsu, T. Tateno, H.P.C. Robinson, A system for MEA-based multisite stimulation, IEEE Trans. Biomed. Eng. 50 (2003) 241-248.

[22] N. Joye, A. Schmid, Y. Leblebici, Three-dimensional silicon-based MEA with high spatial resolution, in: Proceedings of the Sixth International Meeting on Substrate-Integrated Microelectrodes, Reutlingen, Germany, 2008, pp. 310-311.
[23] N. Joye, A. Schmid, Y. Leblebici, An electrical model of the cell-electrode interface for high-density microelectrode arrays, in: Proceedings of the 30th Annual International IEEE EMBS Conference, 2008, pp. 559-562.

[24] N. Joye, A. Schmid, Y. Leblebici, A cell-electrode interface model for highdensity microelectrode arrays, in: Proceedings of the 31th Annual International IEEE EMBS Conference, 2009, pp. 3247-3250.

[25] G.T.A. Kovacs, Introduction to the theory, design, and modeling of thin-film microelectrodes for neural interfaces, in: D.A. Stenger, T.M. McKenna (Eds.), Enabling Technologies for Cultured Neural Networks, Academic Press, London, 1994, pp. 121-166.

[26] S. Marom, G. Shahaf, Development, learning and memory in large random networks of cortical neurons: lessons beyond anatomy, Q. Rev. Biophys. 35 (2002) 63-87.

[27] P. Massobrio, G. Massobrio, S. Martinoia, Multi-program approach for simulating recorded extracellular signals generated by neurons coupled to microelectrode arrays, Neurocomputing 70 (2007) 2467-2476.

[28] A. Mazzatenta, M. Giugliano, S. Campidelli, L. Gambazzi, L. Businaro, H. Markram, M. Prato, L. Ballerini, Interfacing neurons with carbon nanotubes: electrical signal transfer and synaptic stimulation in cultured brain circuits, J. Neurosci. 27 (2007) 6931-6936.

[29] J. Newman, Resistance for flow of current to a disk, J. Electrochem. Soc. 113 (1966) 501-502.

[30] R.H. Olsson III, D.L. Buhl, A.M. Sirota, G. Buzsaki, K.D. Wise, Band-tunable and multiplexed integrated circuits for simultaneous recording and stimulation with microelectrode arrays, IEEE Trans. Biomed. Eng. 52 (2005) 1303-1311.

[31] R. Plonsey, R.C. Barr, Bioelectricity: A Quantitative Approach, Springer, New York, 2007.

[32] A. Richardot, E.T. McAdams, Harmonic analysis of low-frequency bioelectrode behavior, IEEE Trans. Med. Imaging 21 (2002) 604-612.

[33] W.L.C. Rutten, Selective electrical interfaces with the nervous system, Annu. Rev. Biomed. Eng. 4 (2002) 407-452.

[34] I. Schoen, P. Fromherz, The mechanism of extracellular stimulation of nerve cells on an electrolyte-oxide-semiconductor capacitor, Biophys. J. 92 (2007) 1096-1111.

[35] M. Schuettler, Electrochemical properties of platinum electrodes in vitro: comparison of six different surface qualities, in: Proceedings of the 29th Annual International IEEE EMBS Conference, 2007, pp. 186-189.

[36] D.A. Sverjensky, Interpretation and prediction of triple-layer model capacitances and the structure of the oxide-electrolyte-water interface, Geochimica et Cosmochimica Acta 65 (2001) 3643-3655.

[37] P. Thiébaud, C. Beuret, M. Koudelka-Hep, M. Bove, S. Martinoia, M. Grattarola, H. Jahnsen, R. Rebaudo, M. Balestrino, J. Zimmer, Y. Dupont, An array of Pt-tip microelectrodes for extracellular monitoring of activity of brain slices, Biosens. Bioelectron. 14 (1999) 61-65.

[38] W. Wattanapanitch, M. Fee, R. Sarpeshkar, An energy-efficient micropower neural recording amplifier, IEEE Trans. Biomed. Circuits Syst. 1 (2007) 136-147.

[39] R. Weiss, P. Fromherz, Frequency dependent signal transfer in neuron transistors, Phys. Rev. E 55 (1997) 877-889.

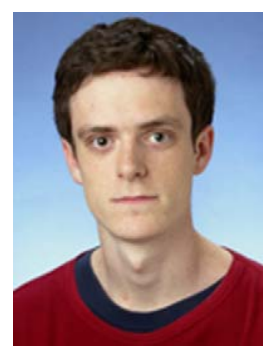

Neil Joye received the M.S degree in Electrical Engineering from the Ecole Polytechnique Fédérale de Lausanne (EPFL), Switzerland, in 2005. He is now working toward the Ph.D. degree in the same institution. His thesis involves the design of high resolution silicon-based microelectrode arrays for electrophysiological experiments.

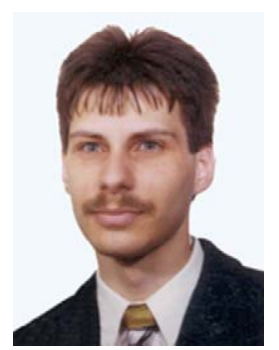

Alexandre Schmid received the M.S. degree in Microengineering and the Ph.D. degree in Electrical Engineering from the Swiss Federal Institute of Technology (EPFL) in 1994 and 2000, respectively. He has been with the EPFL since 1994, working at the Integrated Systems Laboratory as a Research Assistant, and at the Electronics Laboratories as a post-doctoral fellow. His earlier work included high-speed video data compression, CAD for high-performance telecommunication architectures, hardware implementation of artificial neural networks and digital decimation filters for high-speed delta-sigma converters. He joined the Microelectronic Systems Laboratory in 2002 as a Senior Research Associate, where he has been conducting research in the field of nonconventional signal processing hardware, nanoelectronic reliability, and bioelectronic interfaces. Since 2004, Dr. Schmid is also teaching at the Microengineering and Electrical Engineering Departments of EPFL. 


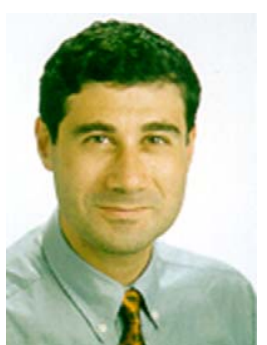

Yusuf Leblebici received the B.S. and M.S. degrees in Electrical Engineering from Istanbul Technical University in 1984 and 1986, respectively, and the Ph.D. degree in Electrical and Computer Engineering from the University of Illinois at Urbana-Champaign (UIUC) in 1990. Between 1991 and 2001, he worked as a faculty member at UIUC, at Istanbul Technical University, and at Worcester Polytechnic Institute (WPI) where he established and directed the VLS

Design Laboratory, and also served as a project director at the New England Center for Analog and Mixed-Signal IC Design. Since January 2002, Dr. Leblebici has been a Chair Professor at the Swis Federal Institute of Technology in Lausanne (EPFL), and director of the Microelectronic Systems Laboratory. His research interests include design of high-speed CMOS digital and mixed-signal integrated circuits, computer-aided design of VLSI systems, modeling and simulation of nano-electronic circuits, intelligent sensor interfaces, and VLSI reliability analysis. Dr. Leblebici is the coauthor of four textbooks, "Hot-Carrier Reliability of MOS VLSI Circuits" (Kluwer Academic Publishers, 1993), "CMOS Digital Integrated Circuits: Analysis and Design" (McGraw Hill 1st Edition, 1996, 2nd Edition 1999 3rd Edition 2003), "CMOS Multichannel Single-Chip Receivers for Multi-Gigabit Optical Data Communications" (Springer, 2007) and "Fundamentals of HighFrequency CMOS Analog Integrated Circuits" (Cambridge University Press, 2009), as well as more than 150 scientific articles published in international journals and conferences. Dr. Leblebici served on the organizing and steering committees of several international conferences in microelectronics, and he has been the Co-Chairman of the Joint 2006 European Solid-State Circuits Conference/European Solid-State Device Research Conference (ESSCIRC/ESSDERC). He was an Associate Editor of IEEE Transactions on Circuits and Systems II between 1998 and 2000, and an Associate Editor of IEEE Transactions on VLSI between 2001 and 2003. Dr. Leblebici has received the Young Scientist Award of the Turkish Scientific and Technological Research Council in 1995, and the Joseph Samuel Satin Distinguished Fellow Award of the Worcester Polytechnic Institute in 1999. 